CRYSTALLOGRAPHIC COMMUNICATIONS

ISSN 2056-9890

Received 14 November 2016

Accepted 8 December 2016

Edited by A. J. Lough, University of Toronto, Canada

Keywords: crystal structure; thiosemicarbazide cyclization; triazolopyrimidines; Dimroth rearrangement.

CCDC reference: 1521445

Supporting information: this article has supporting information at journals.iucr.org/e

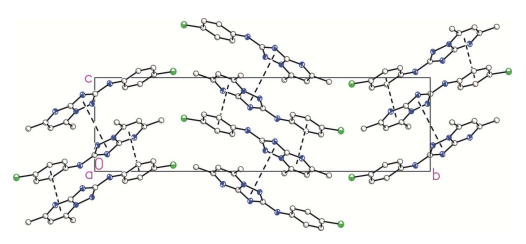

OPEN $\odot$ ACCESS

\section{Synthesis and crystal structure of $\mathrm{N}$-(4-chloro- phenyl)-5,7-dimethyl-1,2,4-triazolo[1,5-a]- pyrimidin-2-amine}

\author{
Hlib Repich, ${ }^{\text {a* }}$ Svitlana Orysyk, ${ }^{a}$ Pavlo Savytskyi $^{\mathrm{b}}$ and Vasyl Pekhnyo ${ }^{\mathrm{a}}$
}

\begin{abstract}
${ }^{a}$ Vernadsky Institute of General and Inorganic Chemistry of the Ukrainian National Academy of Sciences, Palladin av. 32/ 34, 03142 Kyiv, Ukraine, and ${ }^{\mathbf{b}}$ The Institute of Molecular Biology and Genetics of the Ukrainian National Academy of Sciences, Zabolotnogo Str. 150, 03680 Kyiv, Ukraine. *Correspondence e-mail: glebrepich@gmail.com
\end{abstract}

The title compound, $\mathrm{C}_{13} \mathrm{H}_{12} \mathrm{ClN}_{5}$, was synthesized by the cyclization of 1-(4,6dimethylpyrimidin-2-yl)-4-phenylthiosemicarbazide in the presence of $\mathrm{Ni}\left(\mathrm{NO}_{3}\right)_{2}$. The molecular structure of the compound is essentially planar. In the crystal, molecules form dimers via pairs of $\mathrm{N}-\mathrm{H} \cdots \mathrm{N}$ hydrogen bonds between the $\mathrm{H}$ atom of the exocyclic amino group and the $\mathrm{N}$ atom at the 4position of the triazole ring. The resulting dimers are packed into layers which are connected by $\pi$-stacking interactions between the aromatic systems of the pyrimidine and benzene nuclei, and between the triazole cores.

\section{Chemical context}

It is well known that thermal cyclization of 1-(pyrymidin-2yl)thiosemicarbazides leads to the formation of mercapto derivatives of triazolopyrimidine (Babichev \& Kovtunenko, 1977; Kottke \& Kuhmshtedt, 1978). In contrast to this, it has been shown that analogous substrates can be converted into the corresponding 2 - $R$-amino-5,7-dimethyl $[1,2,4]$ triazolo[1,5-a]pyrimidines by cyclization in the presence of methyl iodide and sodium acetate in boiling ethanol solution. Such processes undergo alcylation of a sulfur atom with the formation of the $S$-methyl derivative, which then undergoes intramolecular cyclization with elimination of a methanethiol molecule and the formation of the unstable intermediate $A$. The subsequent Dimroth rearrangement of intermediate $A$ gives the final product $B$ (Fig. 1) (Vas'kevich et al., 2006). In the present work we show that an analogous cyclization followed by Dimroth rearrangement can proceed in mild conditions in the presence of $\mathrm{Ni}^{2+}$ ions (Fig. 1).

\section{Structural commentary}

The molecular structure of the title compound is almost planar. The molecule consists of two flat fragments: the

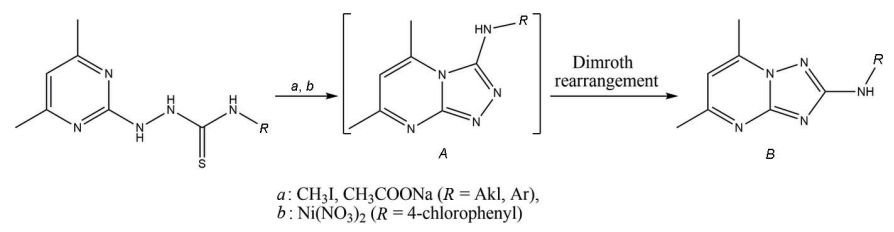

Figure 1

Scheme showing the formation of related compounds $(a)$ according to the literature and $(b)$ in the present work. 
$[1,2,4]$ triazolo[1,5-a]pyrimidine moiety, and the 4-chlorophenyl group. The mean deviation from the N1/C2/C3/C4/N2/ $\mathrm{C} 6 / \mathrm{N} 3 / \mathrm{C} 7 / \mathrm{N} 4$ plane is $0.010 \AA$ while that from the $\mathrm{C} 8-\mathrm{C} 13$ plane is $0.006 \AA$. The dihedral angle between these planes is $6.23(5)^{\circ}$. The sum of the $\mathrm{C} 7-\mathrm{N} 5-\mathrm{C} 8, \mathrm{C} 7-\mathrm{N} 5-\mathrm{H} 1$ and $\mathrm{C} 8-\mathrm{N} 5-\mathrm{H} 1$ angles is $359.86^{\circ}$, indicating $s p^{2}$ hybridization of atom N5.

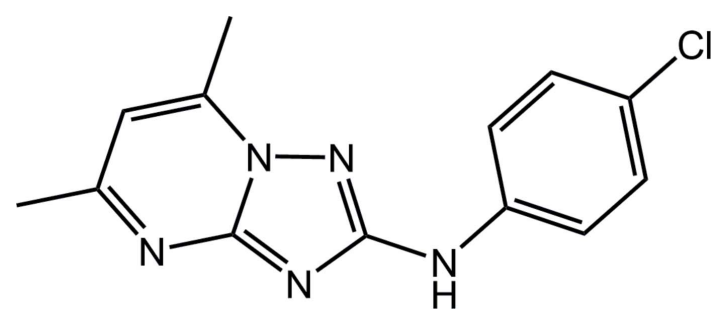

\section{Supramolecular features}

In the crystal, molecules form inversion dimers via pairs of $\mathrm{N} 5-\mathrm{H} 1 \cdots \mathrm{N} 3^{\mathrm{i}}$ hydrogen bonds (Table 1, Fig. 2). The resulting dimers are packed into layers parallel to the $b c$ plane. These layers are connected by $\pi$-stacking interactions between the aromatic systems of the pyrimidine and benzene rings, and between triazole cores (Figs. 3 and 4). The centroid-centroid distance between the benzene ring of the 4-chlorophenyl group $(\mathrm{C} 8-\mathrm{C} 13)$ and the pyrimidine ring $(\mathrm{N} 1 / \mathrm{C} 2 / \mathrm{C} 3 / \mathrm{C} 4 / \mathrm{N} 2 /$ C6) of symmetry-related molecules is 3.513 (1) $\AA$. These overlapping rings have a slip angle of $16.3^{\circ}$. The centroidcentroid distance between five-membered (N1/N4/C7/N3/C6) triazole rings is 3.824 (1) $\AA$ with a slip angle of $29.0^{\circ}$.

In general, the crystal structure of the title compound is very similar to that of 5,7-dimethyl-2-phenylamino-1,2,4-triazolo[1,5-a]pyrimidine (Vas'kevich et al., 2006).

\section{Synthesis and crystallization}

A warm solution of $\mathrm{Ni}\left(\mathrm{NO}_{3}\right)_{2}(0.0364 \mathrm{~g}, 0.125 \mathrm{mmol}$ in $15 \mathrm{ml}$ of ethanol) was added dropwise under vigorous stirring to a warm solution of 1-(4,6-dimethylpyrimidin-2-yl)-4-phenylthiosemicarbazide $(0.0767 \mathrm{~g}, 0.25 \mathrm{mmol}$ in $20 \mathrm{ml}$ of ethanol), prepared according to a known procedure (Vas'kevich et al.,

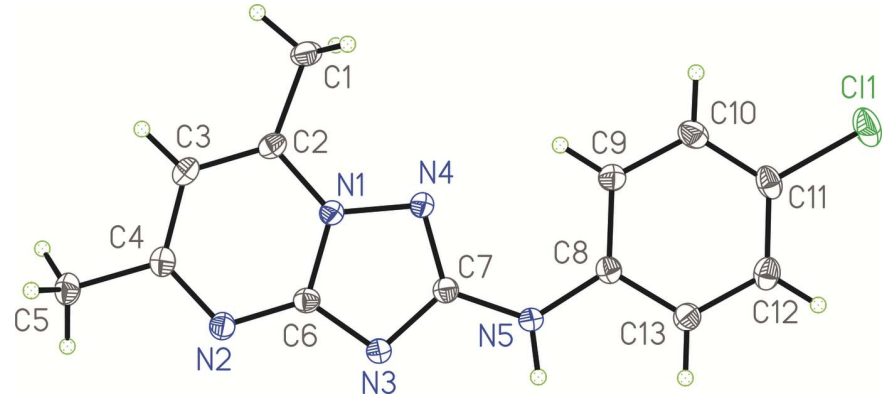

Figure 2

The molecular structure of the title compound. Displacement ellipsoids are drawn at the $50 \%$ probability level.
Table 1

Hydrogen-bond geometry $\left(\AA{ }^{\circ}\right)$.

\begin{tabular}{lllll}
\hline$D-\mathrm{H} \cdots A$ & $D-\mathrm{H}$ & $\mathrm{H} \cdots A$ & $D \cdots A$ & $D-\mathrm{H} \cdots A$ \\
\hline $\mathrm{N} 5-\mathrm{H} 1 \cdots \mathrm{N} 3^{\mathrm{i}}$ & $0.870(18)$ & $2.109(18)$ & $2.9748(14)$ & $173.5(16)$ \\
\hline
\end{tabular}

Symmetry code: (i) $-x+1,-y,-z+2$.

2006). An orange precipitate of the $\mathrm{Ni}^{2+}$ complex $(M: L=1: 2)$ was formed. The resulting mixture was left for a few days. Detailed analysis of the obtained compound showed the presence of a significant amount of colourless plate-shaped crystals of the title compound, which were used for X-ray analysis.

\section{Refinement}

Crystal data, data collection and structure refinement details are summarized in Table 2. All $\mathrm{H}$ atoms bonded to $\mathrm{C}$ atoms were placed in geometrically idealized positions according to hybridization and constrained to ride on their parent $\mathrm{C}$ atoms, with $\mathrm{C}-\mathrm{H}$ bonds for the aromatic rings and methyl groups of 0.95 and $0.98 \AA$, respectively, with $U_{\text {iso }}\left(\mathrm{H}_{\text {aromatic }}\right)=1.2 U_{\text {eq }}(\mathrm{C})$ and $U_{\text {iso }}\left(\mathrm{H}_{\text {methyl }}\right)=1.5 U_{\text {eq }}(\mathrm{C})$. The methyl groups were allowed to rotate freely about the $\mathrm{C}-\mathrm{C}$ bonds. The $\mathrm{H}$ atom bonded to the $\mathrm{N}$ atom was located in a difference map and refined without any restraints.

Table 2

Experimental details.

Crystal data
Chemical formula

$M_{\mathrm{r}}$

Crystal system, space group

Temperature $(\mathrm{K})$

$a, b, c(\AA)$

$\beta\left({ }^{\circ}\right)$

$V\left(\AA^{3}\right)$

Z

Radiation type

$\mu\left(\mathrm{mm}^{-1}\right)$

Crystal size $(\mathrm{mm})$

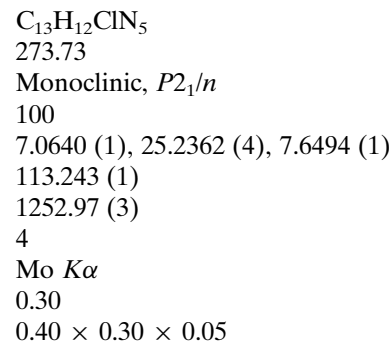

Data collection

Diffractometer

Absorption correction

$T_{\text {min }}, T_{\text {max }}$

No. of measured, independent and observed $[I>2 \sigma(I)]$ reflections

$R_{\text {int }}$

$(\sin \theta / \lambda)_{\max }\left(\AA^{-1}\right)$

Bruker APEXII CCD

Multi-scan ( $S A D A B S$; Bruker, 2001)

$0.874,0.985$

$11572,3837,3347$

0.018

0.716

Refinement

$R\left[F^{2}>2 \sigma\left(F^{2}\right)\right], w R\left(F^{2}\right), S \quad 0.039,0.100,1.04$

No. of reflections

No. of parameters

$\mathrm{H}$-atom treatment

$\Delta \rho_{\max }, \Delta \rho_{\min }\left(\mathrm{e} \AA^{-3}\right)$
3837

178

$\mathrm{H}$ atoms treated by a mixture of independent and constrained refinement

$0.47,-0.33$
Computer programs: APEX2 and SAINT (Bruker, 2007), SHELXS97 and SHELXTL (Sheldrick, 2008), SHELXL2014 (Sheldrick, 2015) and publCIF (Westrip, 2010). 


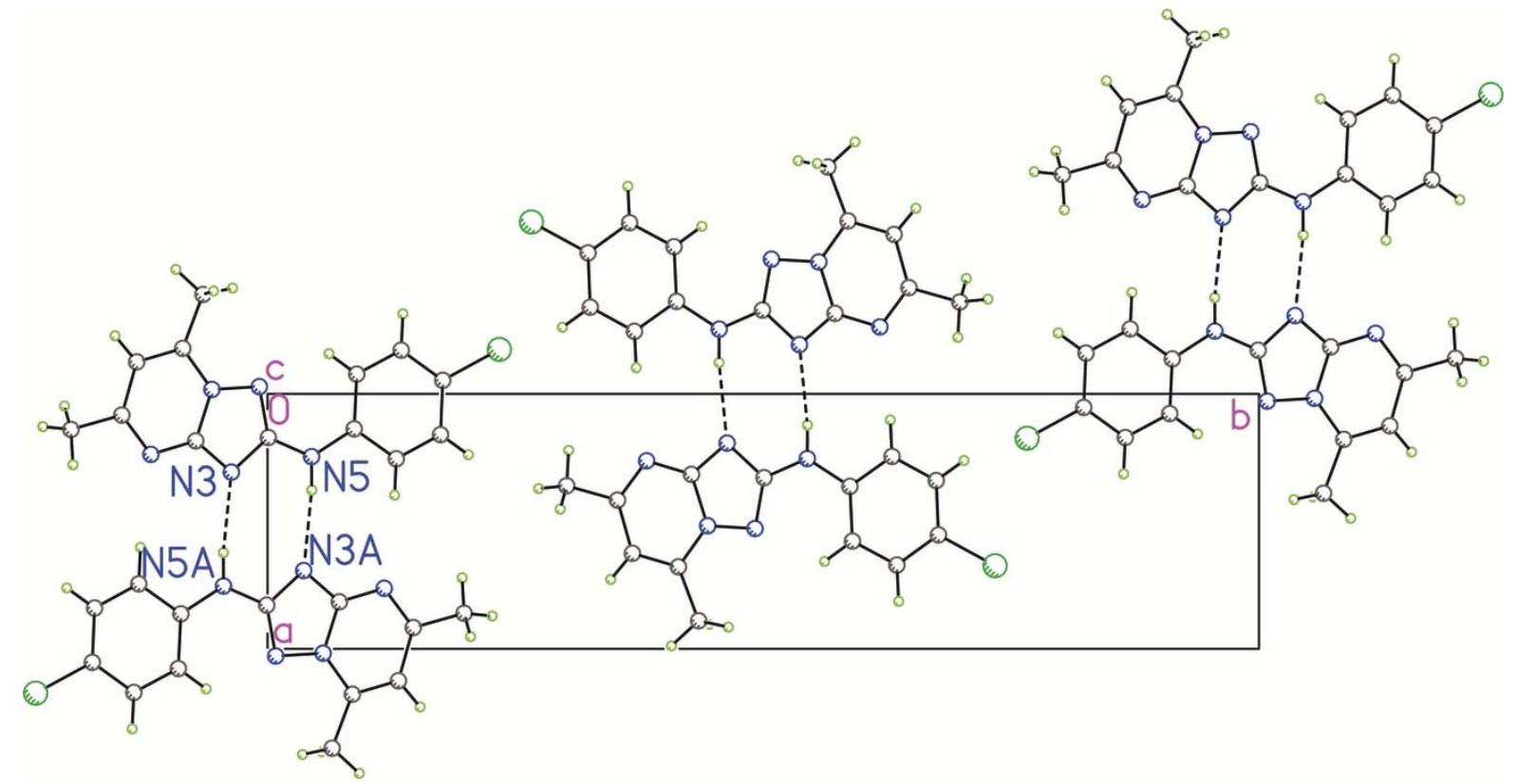

Figure 3

Packing diagram of the title compound with $\mathrm{N}-\mathrm{H} \cdots \mathrm{N}$ hydrogen bonds shown as dashed lines. The projection is shown along [001] and the atoms labelled with suffix $A$ are related by an inversion centre (symmetry code $1-x,-y, 2-z$ ).

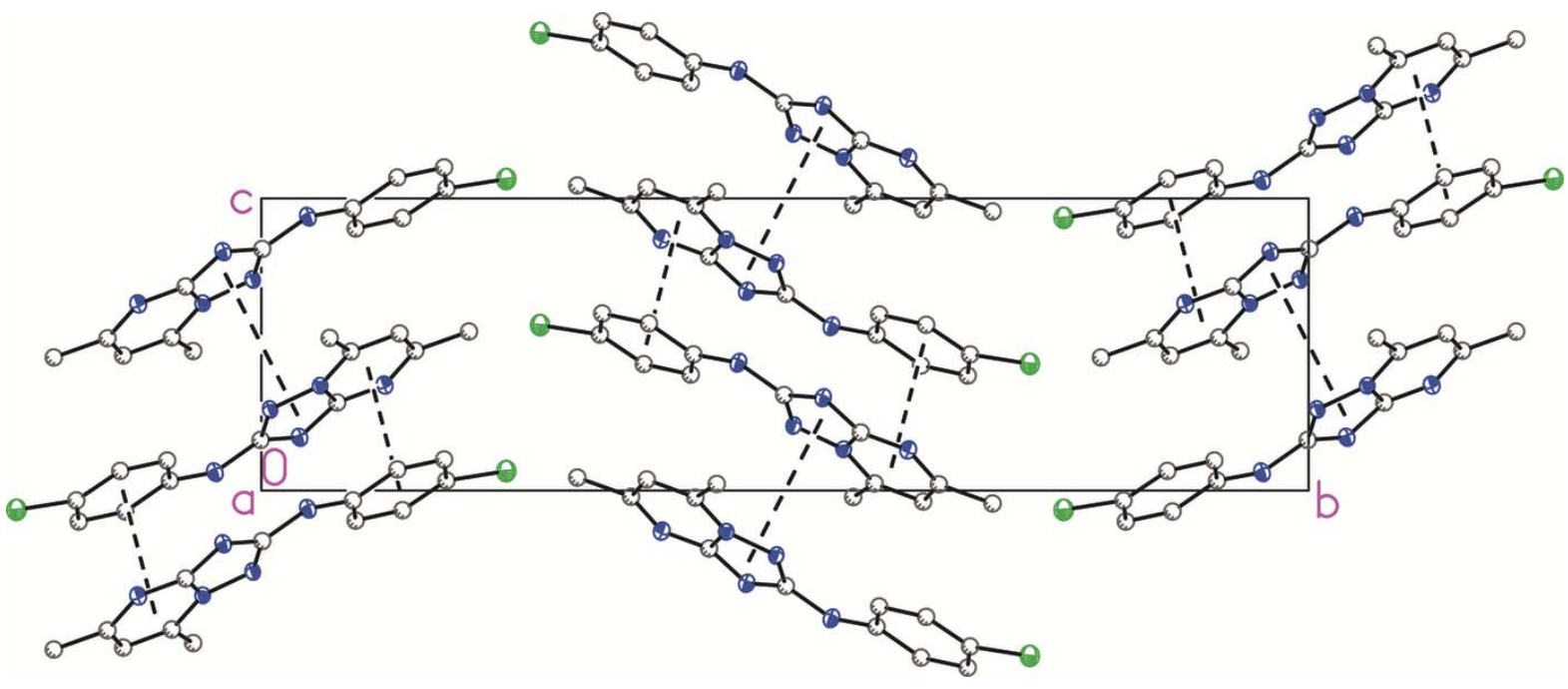

Figure 4

Packing diagram of the title compound with $\pi-\pi$ interactions between aromatic systems represented by dashed lines. The projection is shown along [100]. $\mathrm{H}$ atoms have been omitted for clarity.

\section{Acknowledgements}

The authors thank the Ukrainian Academy of Sciences for financial support.

\section{References}

Babichev, F. S. \& Kovtunenko, V. A. (1977). Chem. Heterocycl. Compd. 13, 117-131.
Bruker (2001). SADABS. Bruker AXS Inc., Madison, Wisconsin, USA.

Bruker (2007). APEX2 and SAINT. Bruker AXS Inc., Madison, Wisconsin, USA.

Kottke, K. \& Kuhmshtedt, K. (1978). Pharmazie, 33, 124-125.

Sheldrick, G. M. (2008). Acta Cryst. A64, 112-122.

Sheldrick, G. M. (2015). Acta Cryst. C71, 3-8.

Vas'kevich, R. I., Savitskii, P. V., Zborovskii, Yu. L., Staninets, V. I., Rusanov, E. B. \& Chernega, A. N. (2006). Russ. J. Org. Chem. 42, 1403-1408.

Westrip, S. P. (2010). J. Appl. Cryst. 43, 920-925. 


\section{supporting information}

Acta Cryst. (2017). E73, 35-37 [https://doi.org/10.1107/S2056989016019629]

Synthesis and crystal structure of $\mathrm{N}$-(4-chlorophenyl)-5,7-dimethyl-1,2,4triazolo[1,5-a]pyrimidin-2-amine

Hlib Repich, Svitlana Orysyk, Pavlo Savytskyi and Vasyl Pekhnyo

Computing details

Data collection: APEX2 (Bruker, 2007); cell refinement: SAINT (Bruker, 2007); data reduction: SAINT (Bruker, 2007); program(s) used to solve structure: SHELXS97 (Sheldrick, 2008); program(s) used to refine structure: SHELXL2014 (Sheldrick, 2015); molecular graphics: SHELXTL (Sheldrick, 2008); software used to prepare material for publication: SHELXTL (Sheldrick, 2008) and publCIF (Westrip, 2010).

N-(4-Chlorophenyl)-5,7-dimethyl-1,2,4-triazolo[1,5-a]pyrimidin-2-amine

Crystal data

$\mathrm{C}_{13} \mathrm{H}_{12} \mathrm{ClN}_{5}$

$M_{r}=273.73$

Monoclinic, $P 2{ }_{1} / n$

$a=7.0640(1) \AA$

$b=25.2362(4) \AA$

$c=7.6494(1) \AA$

$\beta=113.243(1)^{\circ}$

$V=1252.97(3) \AA^{3}$

$Z=4$

Data collection

Bruker APEXII CCD diffractometer

Radiation source: sealed tube $\varphi$ and $\omega$ scans

Absorption correction: multi-scan

(SADABS; Bruker, 2001)

$T_{\min }=0.874, T_{\max }=0.985$

11572 measured reflections

\section{Refinement}

Refinement on $F^{2}$

Least-squares matrix: full

$R\left[F^{2}>2 \sigma\left(F^{2}\right)\right]=0.039$

$w R\left(F^{2}\right)=0.100$

$S=1.04$

3837 reflections

178 parameters

0 restraints

Primary atom site location: structure-invariant direct methods
$F(000)=568$

$D_{\mathrm{x}}=1.451 \mathrm{Mg} \mathrm{m}^{-3}$

Mo $K \alpha$ radiation, $\lambda=0.71073 \AA$

Cell parameters from 5527 reflections

$\theta=3.0-30.5^{\circ}$

$\mu=0.30 \mathrm{~mm}^{-1}$

$T=100 \mathrm{~K}$

Plate, colorless

$0.40 \times 0.30 \times 0.05 \mathrm{~mm}$

3837 independent reflections

3347 reflections with $I>2 \sigma(I)$

$R_{\text {int }}=0.018$

$\theta_{\max }=30.6^{\circ}, \theta_{\min }=1.6^{\circ}$

$h=-10 \rightarrow 8$

$k=-36 \rightarrow 33$

$l=-6 \rightarrow 10$

Secondary atom site location: difference Fourier map

Hydrogen site location: mixed

$\mathrm{H}$ atoms treated by a mixture of independent and constrained refinement

$w=1 /\left[\sigma^{2}\left(F_{\mathrm{o}}^{2}\right)+(0.047 P)^{2}+0.6408 P\right]$ where $P=\left(F_{\mathrm{o}}^{2}+2 F_{\mathrm{c}}^{2}\right) / 3$

$(\Delta / \sigma)_{\max }=0.001$

$\Delta \rho_{\max }=0.47 \mathrm{e} \AA^{-3}$

$\Delta \rho_{\min }=-0.33$ e $\AA^{-3}$ 


\section{Special details}

Geometry. All esds (except the esd in the dihedral angle between two 1.s. planes) are estimated using the full covariance matrix. The cell esds are taken into account individually in the estimation of esds in distances, angles and torsion angles; correlations between esds in cell parameters are only used when they are defined by crystal symmetry. An approximate (isotropic) treatment of cell esds is used for estimating esds involving l.s. planes.

Refinement. Refinement of $F^{2}$ against ALL reflections. The weighted $R$-factor $w R$ and goodness of fit $S$ are based on $F^{2}$, conventional $R$-factors $R$ are based on $F$, with $F$ set to zero for negative $F^{2}$. The threshold expression of $F^{2}>\sigma\left(F^{2}\right)$ is used only for calculating $R$-factors (gt) etc. and is not relevant to the choice of reflections for refinement. $R$-factors based on $F^{2}$ are statistically about twice as large as those based on $F$, and $R$ - factors based on ALL data will be even larger.

Fractional atomic coordinates and isotropic or equivalent isotropic displacement parameters $\left(\AA^{2}\right)$

\begin{tabular}{|c|c|c|c|c|}
\hline & $x$ & $y$ & $z$ & $U_{\text {iso }} * / U_{\text {eq }}$ \\
\hline $\mathrm{C} 1$ & $-0.39001(18)$ & $-0.06515(5)$ & 0.47760 (19) & $0.0212(2)$ \\
\hline H1A & -0.4039 & -0.0542 & 0.5949 & $0.032 *$ \\
\hline H1B & -0.4903 & -0.0931 & 0.4153 & $0.032 *$ \\
\hline $\mathrm{H} 1 \mathrm{C}$ & -0.4154 & -0.0347 & 0.3919 & $0.032 *$ \\
\hline $\mathrm{C} 2$ & $-0.17835(18)$ & $-0.08555(5)$ & $0.52419(16)$ & $0.0163(2)$ \\
\hline $\mathrm{C} 3$ & $-0.12544(19)$ & $-0.13199(5)$ & $0.46223(17)$ & $0.0176(2)$ \\
\hline $\mathrm{H} 3$ & -0.2303 & -0.1544 & 0.3786 & $0.021^{*}$ \\
\hline $\mathrm{C} 4$ & $0.08323(19)$ & $-0.14699(5)$ & $0.52120(17)$ & $0.0171(2)$ \\
\hline $\mathrm{C} 5$ & $0.1395(2)$ & $-0.19827(5)$ & 0.45558 (19) & $0.0212(2)$ \\
\hline $\mathrm{H} 5 \mathrm{C}$ & 0.2813 & -0.1962 & 0.4637 & $0.032 *$ \\
\hline H5B & 0.0457 & -0.2050 & 0.3235 & $0.032 *$ \\
\hline $\mathrm{H} 5 \mathrm{~A}$ & 0.1285 & -0.2272 & 0.5366 & $0.032 *$ \\
\hline C6 & $0.18434(17)$ & $-0.07241(4)$ & $0.69865(16)$ & $0.0154(2)$ \\
\hline $\mathrm{C} 7$ & $0.17125(17)$ & $0.00081(4)$ & $0.82697(16)$ & $0.0152(2)$ \\
\hline $\mathrm{C} 8$ & $0.13730(18)$ & $0.08741(4)$ & $0.96620(16)$ & $0.0156(2)$ \\
\hline C9 & $-0.07753(18)$ & $0.08999(5)$ & $0.89705(17)$ & $0.0179(2)$ \\
\hline H9 & -0.1590 & 0.0613 & 0.8262 & $0.022 *$ \\
\hline $\mathrm{C} 10$ & $-0.17128(19)$ & $0.13482(5)$ & $0.93266(18)$ & $0.0203(2)$ \\
\hline H10 & -0.3172 & 0.1366 & 0.8871 & $0.024^{*}$ \\
\hline C11 & $-0.0532(2)$ & $0.17674(5)$ & $1.03401(17)$ & $0.0199(2)$ \\
\hline $\mathrm{C} 12$ & $0.1601(2)$ & $0.17448(5)$ & $1.10631(18)$ & $0.0202(2)$ \\
\hline H12 & 0.2404 & 0.2031 & 1.1785 & $0.024^{*}$ \\
\hline $\mathrm{C} 13$ & 0.25465 (19) & $0.12990(5)$ & $1.07201(17)$ & $0.0184(2)$ \\
\hline H13 & 0.4008 & 0.1281 & 1.1208 & $0.022 *$ \\
\hline $\mathrm{Cl1}$ & $-0.17415(6)$ & $0.23384(2)$ & $1.06639(5)$ & $0.02889(10)$ \\
\hline H1 & $0.378(3)$ & $0.0447(7)$ & $1.006(2)$ & $0.023(4)^{*}$ \\
\hline N1 & $-0.01741(15)$ & -0.05610 & $0.64112(14)$ & $0.01484(19)$ \\
\hline N3 & $0.30697(15)$ & -0.03648 & $0.81768(15)$ & $0.01627(19)$ \\
\hline N5 & $0.24606(16)$ & $0.04442(4)$ & $0.93749(15)$ & $0.0171(2)$ \\
\hline N4 & $-0.02837(15)$ & -0.00828 & $0.72245(14)$ & $0.01575(19)$ \\
\hline $\mathrm{N} 2$ & $0.23744(16)$ & $-0.11766(4)$ & $0.63896(15)$ & $0.0173(2)$ \\
\hline
\end{tabular}

Atomic displacement parameters $\left(\AA^{2}\right)$

\begin{tabular}{lllllll}
\hline & $U^{11}$ & $U^{22}$ & $U^{33}$ & $U^{12}$ & $U^{13}$ & $U^{23}$ \\
\hline $\mathrm{C} 1$ & $0.0139(5)$ & $0.0218(6)$ & $0.0243(6)$ & $-0.0013(4)$ & $0.0036(5)$ & $0.0008(5)$
\end{tabular}




$\begin{array}{lllllll}\text { C2 } & 0.0154(5) & 0.0171(5) & 0.0145(5) & -0.0025(4) & 0.0038(4) & 0.0020(4) \\ \text { C3 } & 0.0183(5) & 0.0169(5) & 0.0161(5) & -0.0041(4) & 0.0050(4) & -0.0006(4) \\ \text { C4 } & 0.0210(5) & 0.0155(5) & 0.0163(5) & -0.0013(4) & 0.0090(4) & 0.0000(4) \\ \text { C5 } & 0.0253(6) & 0.0178(5) & 0.0220(6) & -0.0013(4) & 0.0109(5) & -0.0046(4) \\ \text { C6 } & 0.0144(5) & 0.0160(5) & 0.0161(5) & -0.0008(4) & 0.0064(4) & 0.0012(4) \\ \text { C7 } & 0.0153(5) & 0.0147(5) & 0.0156(5) & -0.0002(4) & 0.0060(4) & 0.0006(4) \\ \text { C8 } & 0.0176(5) & 0.0146(5) & 0.0144(5) & 0.0018(4) & 0.0063(4) & 0.0017(4) \\ \text { C9 } & 0.0168(5) & 0.0180(5) & 0.0178(5) & 0.0006(4) & 0.0056(4) & -0.0002(4) \\ \text { C10 } & 0.0186(5) & 0.0223(6) & 0.0192(6) & 0.0053(4) & 0.0068(5) & 0.0024(4) \\ \text { C11 } & 0.0271(6) & 0.0167(5) & 0.0166(5) & 0.0073(4) & 0.0092(5) & 0.0026(4) \\ \text { C12 } & 0.0264(6) & 0.0156(5) & 0.0174(5) & 0.0003(4) & 0.0074(5) & 0.0006(4) \\ \text { C13 } & 0.0189(5) & 0.0162(5) & 0.0184(5) & 0.0002(4) & 0.0056(4) & 0.0006(4) \\ \text { C11 } & 0.03686(19) & 0.02176(16) & 0.02714(18) & 0.01310(12) & 0.01167(14) & 0.00019(12) \\ \text { N1 } & 0.0142(4) & 0.0138(4) & 0.0160(5) & -0.0005(3) & 0.0053(4) & -0.0002(3) \\ \text { N3 } & 0.0140(4) & 0.0153(4) & 0.0190(5) & -0.0003(3) & 0.0061(4) & -0.0019(4) \\ \text { N5 } & 0.0128(4) & 0.0158(4) & 0.0203(5) & 0.0002(3) & 0.0040(4) & -0.0031(4) \\ \text { N4 } & 0.0149(4) & 0.0134(4) & 0.0175(5) & -0.0003(3) & 0.0050(4) & -0.0013(3) \\ \text { N2 } & 0.0177(5) & 0.0161(4) & 0.0192(5) & -0.0011(4) & 0.0084(4) & -0.0015(4)\end{array}$

Geometric parameters $\left(\AA,{ }^{\circ}\right)$

\begin{tabular}{|c|c|c|c|}
\hline $\mathrm{C} 1-\mathrm{C} 2$ & $1.4857(16)$ & $\mathrm{C} 7-\mathrm{N} 5$ & $1.3612(15)$ \\
\hline $\mathrm{C} 1-\mathrm{H} 1 \mathrm{~A}$ & 0.9800 & $\mathrm{C} 7-\mathrm{N} 3$ & $1.3651(14)$ \\
\hline $\mathrm{C} 1-\mathrm{H} 1 \mathrm{~B}$ & 0.9800 & $\mathrm{C} 8-\mathrm{N} 5$ & $1.3960(14)$ \\
\hline $\mathrm{C} 1-\mathrm{H} 1 \mathrm{C}$ & 0.9800 & $\mathrm{C} 8-\mathrm{C} 9$ & $1.3977(16)$ \\
\hline $\mathrm{C} 2-\mathrm{N} 1$ & $1.3574(15)$ & $\mathrm{C} 8-\mathrm{C} 13$ & $1.4008(16)$ \\
\hline $\mathrm{C} 2-\mathrm{C} 3$ & $1.3702(16)$ & $\mathrm{C} 9-\mathrm{C} 10$ & $1.3911(16)$ \\
\hline $\mathrm{C} 3-\mathrm{C} 4$ & $1.4124(17)$ & $\mathrm{C} 9-\mathrm{H} 9$ & 0.9500 \\
\hline $\mathrm{C} 3-\mathrm{H} 3$ & 0.9500 & $\mathrm{C} 10-\mathrm{C} 11$ & $1.3805(18)$ \\
\hline $\mathrm{C} 4-\mathrm{N} 2$ & $1.3309(15)$ & $\mathrm{C} 10-\mathrm{H} 10$ & 0.9500 \\
\hline $\mathrm{C} 4-\mathrm{C} 5$ & $1.4976(16)$ & $\mathrm{C} 11-\mathrm{C} 12$ & $1.3859(18)$ \\
\hline $\mathrm{C} 5-\mathrm{H} 5 \mathrm{C}$ & 0.9800 & $\mathrm{C} 11-\mathrm{C} 11$ & $1.7423(12)$ \\
\hline $\mathrm{C} 5-\mathrm{H} 5 \mathrm{~B}$ & 0.9800 & $\mathrm{C} 12-\mathrm{C} 13$ & $1.3855(16)$ \\
\hline $\mathrm{C} 5-\mathrm{H} 5 \mathrm{~A}$ & 0.9800 & $\mathrm{C} 12-\mathrm{H} 12$ & 0.9500 \\
\hline $\mathrm{C} 6-\mathrm{N} 3$ & $1.3338(15)$ & $\mathrm{C} 13-\mathrm{H} 13$ & 0.9500 \\
\hline $\mathrm{C} 6-\mathrm{N} 2$ & $1.3374(15)$ & $\mathrm{N} 1-\mathrm{N} 4$ & $1.3737(13)$ \\
\hline $\mathrm{C} 6-\mathrm{N} 1$ & $1.3781(15)$ & $\mathrm{N} 5-\mathrm{H} 1$ & $0.870(18)$ \\
\hline $\mathrm{C} 7-\mathrm{N} 4$ & $1.3381(15)$ & & \\
\hline $\mathrm{C} 2-\mathrm{C} 1-\mathrm{H} 1 \mathrm{~A}$ & 109.5 & $\mathrm{~N} 5-\mathrm{C} 8-\mathrm{C} 9$ & $124.01(11)$ \\
\hline $\mathrm{C} 2-\mathrm{C} 1-\mathrm{H} 1 \mathrm{~B}$ & 109.5 & $\mathrm{~N} 5-\mathrm{C} 8-\mathrm{C} 13$ & $116.67(11)$ \\
\hline $\mathrm{H} 1 \mathrm{~A}-\mathrm{C} 1-\mathrm{H} 1 \mathrm{~B}$ & 109.5 & $\mathrm{C} 9-\mathrm{C} 8-\mathrm{C} 13$ & $119.32(11)$ \\
\hline $\mathrm{C} 2-\mathrm{C} 1-\mathrm{H} 1 \mathrm{C}$ & 109.5 & $\mathrm{C} 10-\mathrm{C} 9-\mathrm{C} 8$ & $119.56(11)$ \\
\hline $\mathrm{H} 1 \mathrm{~A}-\mathrm{C} 1-\mathrm{H} 1 \mathrm{C}$ & 109.5 & $\mathrm{C} 10-\mathrm{C} 9-\mathrm{H} 9$ & 120.2 \\
\hline $\mathrm{H} 1 \mathrm{~B}-\mathrm{C} 1-\mathrm{H} 1 \mathrm{C}$ & 109.5 & $\mathrm{C} 8-\mathrm{C} 9-\mathrm{H} 9$ & 120.2 \\
\hline $\mathrm{N} 1-\mathrm{C} 2-\mathrm{C} 3$ & $115.11(10)$ & $\mathrm{C} 11-\mathrm{C} 10-\mathrm{C} 9$ & $120.28(11)$ \\
\hline $\mathrm{N} 1-\mathrm{C} 2-\mathrm{C} 1$ & $118.05(11)$ & $\mathrm{C} 11-\mathrm{C} 10-\mathrm{H} 10$ & 119.9 \\
\hline $\mathrm{C} 3-\mathrm{C} 2-\mathrm{C} 1$ & $126.84(11)$ & $\mathrm{C} 9-\mathrm{C} 10-\mathrm{H} 10$ & 119.9 \\
\hline
\end{tabular}




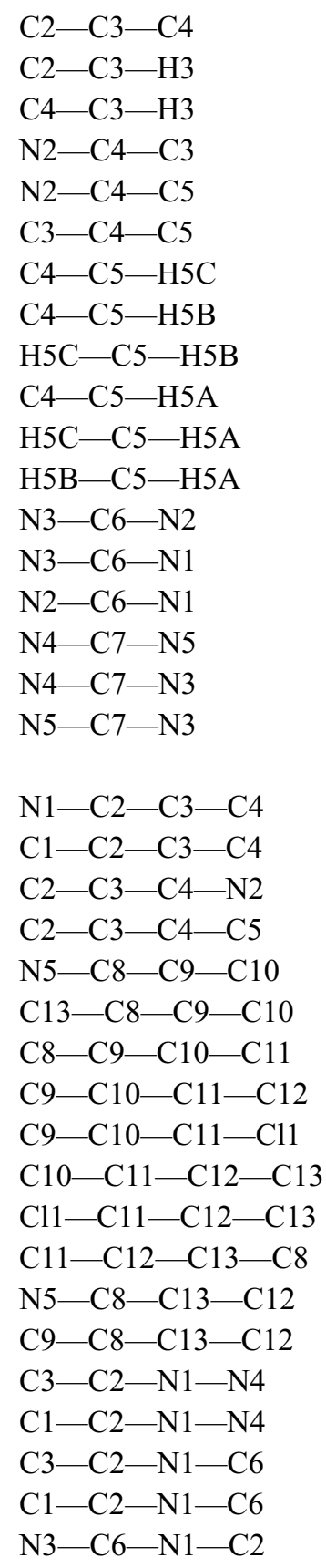

$120.77(11)$

119.6

119.6

$122.65(11)$

$116.91(11)$

$120.42(11)$

109.5

109.5

109.5

109.5

109.5

109.5

$128.28(11)$

$109.03(10)$

$122.69(10)$

$124.68(10)$

$116.54(10)$

$118.77(10)$

$0.80(16)$

$-178.75(11)$

0.44 (18)

$178.90(11)$

$-179.67(11)$

0.64 (17)

$0.64(18)$

$-1.73(19)$

$176.72(9)$

$1.50(18)$

$-176.95(9)$

-0.19 (18)

$179.42(11)$

-0.87 (18)

$-179.95(10)$

$-0.35(17)$

$-2.06(16)$

177.54 (10)

$-177.72(10)$
$\mathrm{C} 10-\mathrm{C} 11-\mathrm{C} 12$

$\mathrm{C} 10-\mathrm{C} 11-\mathrm{C} 11$

$\mathrm{C} 12-\mathrm{C} 11-\mathrm{C} 11$

$\mathrm{C} 11-\mathrm{C} 12-\mathrm{C} 13$

$\mathrm{C} 11-\mathrm{C} 12-\mathrm{H} 12$

$\mathrm{C} 13-\mathrm{C} 12-\mathrm{H} 12$

$\mathrm{C} 12-\mathrm{C} 13-\mathrm{C} 8$

C12-C13-H13

$\mathrm{C} 8-\mathrm{C} 13-\mathrm{H} 13$

$\mathrm{C} 2-\mathrm{N} 1-\mathrm{N} 4$

$\mathrm{C} 2-\mathrm{N} 1-\mathrm{C} 6$

$\mathrm{N} 4-\mathrm{N} 1-\mathrm{C} 6$

$\mathrm{C} 6-\mathrm{N} 3-\mathrm{C} 7$

$\mathrm{C} 7-\mathrm{N} 5-\mathrm{C} 8$

C7-N5-H1

$\mathrm{C} 8-\mathrm{N} 5-\mathrm{H} 1$

C7-N4-N1

$\mathrm{C} 4-\mathrm{N} 2-\mathrm{C} 6$

$\mathrm{N} 2-\mathrm{C} 6-\mathrm{N} 1-\mathrm{C} 2$

N3-C6-N1-N4

$\mathrm{N} 2-\mathrm{C} 6-\mathrm{N} 1-\mathrm{N} 4$

$\mathrm{N} 2-\mathrm{C} 6-\mathrm{N} 3-\mathrm{C} 7$

$\mathrm{N} 1-\mathrm{C} 6-\mathrm{N} 3-\mathrm{C} 7$

$\mathrm{N} 4-\mathrm{C} 7-\mathrm{N} 3-\mathrm{C} 6$

$\mathrm{N} 5-\mathrm{C} 7-\mathrm{N} 3-\mathrm{C} 6$

$\mathrm{N} 4-\mathrm{C} 7-\mathrm{N} 5-\mathrm{C} 8$

N3-C7-N5-C8

$\mathrm{C} 9-\mathrm{C} 8-\mathrm{N} 5-\mathrm{C} 7$

$\mathrm{C} 13-\mathrm{C} 8-\mathrm{N} 5-\mathrm{C} 7$

$\mathrm{N} 5-\mathrm{C} 7-\mathrm{N} 4-\mathrm{N} 1$

$\mathrm{N} 3-\mathrm{C} 7-\mathrm{N} 4-\mathrm{N} 1$

$\mathrm{C} 2-\mathrm{N} 1-\mathrm{N} 4-\mathrm{C} 7$

C6-N1-N4-C7

$\mathrm{C} 3-\mathrm{C} 4-\mathrm{N} 2-\mathrm{C} 6$

$\mathrm{C} 5-\mathrm{C} 4-\mathrm{N} 2-\mathrm{C} 6$

N3- 6 - $-\mathrm{N} 2-\mathrm{C} 4$

$\mathrm{N} 1-\mathrm{C} 6-\mathrm{N} 2-\mathrm{C} 4$
$120.89(11)$

$119.47(10)$

$119.62(10)$

119.16 (11)

120.4

120.4

120.76 (11)

119.6

119.6

$126.67(10)$

$122.58(10)$

110.72 (9)

$102.94(9)$

$128.56(10)$

$116.2(11)$

$115.1(11)$

100.77 (9)

$116.17(10)$

2.19 (17)

0.47 (13)

$-179.62(10)$

$-179.97(12)$

-0.07 (12)

-0.38 (14)

$-179.42(10)$

0.2 (2)

179.17 (11)

6.89 (19)

$-173.41(11)$

179.62 (11)

0.64 (13)

177.46 (11)

-0.64 (12)

-0.44 (17)

$-178.96(10)$

179.07 (11)

-0.82 (16)

Hydrogen-bond geometry $\left(\AA,{ }^{\circ}\right)$

\begin{tabular}{lllll}
\hline$D-\mathrm{H} \cdots A$ & $D-\mathrm{H}$ & $\mathrm{H} \cdots A$ & $D \cdots A$ & $D-\mathrm{H} \cdots A$ \\
\hline $\mathrm{N} 5-\mathrm{H} 1 \cdots \mathrm{N} 3^{\mathrm{i}}$ & $0.870(18)$ & $2.109(18)$ & $2.9748(14)$ & $173.5(16)$ \\
\hline
\end{tabular}

Symmetry code: (i) $-x+1,-y,-z+2$. 